P187 (continued)

Design, Setting and Participants: Fifty undergraduates estimated caloric content for extreme and conventional (e.g., cheeseburger) fair foods and estimated the BMI of people they believed ate these foods. Undergraduates also indicated whether each food should be regulated. To determine the actual BMI of people consuming these foods, researchers coded 2,222 fair attendees as they ate extreme and conventional foods at a U.S. fair. Actual calories were calculated using standard calorie guides.

Outcome Measures and Analysis: Undergraduates' regulation beliefs were predicted from their caloric and BMI estimations. Undergraduates' caloric and BMI estimations were compared with actual values using ANOVAs and chi-squared analyses.

Results: Compared to conventional foods, undergraduates believed extreme foods were $45 \%$ more caloric $(\mathrm{p}<$ $.001)$ and eaten by people with a higher BMI $(\mathrm{p}<.001)$. In actuality, extreme foods were $24 \%$ less caloric than conventional foods, and eaten by people with a lower BMI. Undergraduates' estimations of BMI $(\beta=.593, \mathrm{p}=.001)$ and caloric content $(\beta=.212, \mathrm{p}=.025)$ were significant predictors of regulation beliefs, explaining $31.5 \%$ of the variance; the higher the estimation, the greater the belief. Conclusions and Implications: Beliefs about extreme foods do not correspond with reality. They lead to inaccurate biases and unfounded opinions. It is important to consider these biases when deciding whether to regulate extreme fair foods.

Funding: Cornell Food and Brand Lab.

\section{P188 Health Disparate Score Change Over 12 Months: iCook 4-H Study}

Melissa Olfert, DrPH, RD, melissa.olfert@mail.wvu.edu, West Virginia University, PO Box 6108, Ag. Sc. Building G016, Morgantown, WV 26506-6108; A. Scatterday, MS, Johns Hopkins University; O. Famodu, MS, RD, West Virginia University; J. White, BS; M. Barr, BS;

R. Hagedorn, BS; K. Woehrer, BS; R. Meade, BS, University of Tennessee; $S$. Colby, PhD, RD; L. Franzen-Castle, PhD, RD, University of Nebraska-Lincoln; K. Wilson-Sweebe, MS, South Dakota State University; K. Kattelmann, $P h D, R D$; K. Yerxa, MS, $R D$, University of Maine; $A$. White, $P h D, R D$

Objective: Change in health disparity risk from fall 2013 to fall 2014 was compared in control and treatment groups using a health disparate (HD) score that was first developed in year 1 of the iCook $4-\mathrm{H}$ program. HD was based on 12 variables in a 5-state sample of parent/child dyads $(n=119)$ taking into consideration racial/ethnic status with change variable being used from original definition of HD score.

Design, Setting and Participants: Across five states (ME, NE, SD, TN, WV) research participants including a parent and youth (9-10 y/o when study began) were classified with a HD score using continuous variables from food security questions (6), and two questions about 'running out of food toward the end of the month' and 'if youth eat soon after waking up'. Change of risk over one year was measured across a variable composite score of continuous variables only (8).
Outcome Measures and Analysis: Nested F-tests of a series of linear mixed models and fixed effects with HD as the response and time and group as the predictors.

Results: There is a significant (p-value 0.004) decrease in HD score for time (12 months) but not between group (control or treatment) effect (p-value 0.140) using an additive statistical model.

Conclusions and Implications: Populations that are at risk for health disparity (HD) characteristics may change over time due to learning cooking skills, identifing food resources and planning ahead. It is not understood what 'time' from a skill building program can do to decrease risk of HD but continued data collection will help to further explain this finding.

Funding: USDA.

\section{P189 Blood Pressure and Quality of Life in 9-11 Year Old Youth and Adult Dyad Pairs Over One Year: iCook 4-H}

Melissa Olfert,DrPH, RD, melissa.olfert@mail.wvu.edu, West Virginia University, PO Box 6108, Ag. Sc. Building G016, Morgantown, WV 26506-6108; O. Famodu, MS, RD; J. White, BS; M. Barr, BS; R. Hagedorn, BS; R. Meade, BS, University of Tennessee; $S$. Colby, PhD; L. FranzenCastle, $P h D, R D$, University of Nebraska-Lincoln; T. Aguirre, RN, PhD; C. Kabala, BS, South Dakota State University; K. Kattelmann, $P h D, R D ; D$. Mathews, MS, RD, University of Maine; $A$. White, $P h D, R D$

Objective: To determine if blood pressure (BP) changes and perception of quality of life (PEDS QoL) changes in 9-10 y/o youth and adults after a 12-month culinary skills, family mealtime and physical activity intervention are significant in association using a simple statistical model verses sophisticated analysis.

Design, Setting and Participants: Nested f-tests for child and adults systolic blood pressure (SBP) in group (control $\mathrm{n}=45$; treatment $\mathrm{n}=98$ ); over 12 -month time and PEDS QoL were analyzed. In addition to the two-way analysis an additive model was used. Five states (ME, NE, $\mathrm{SD}, \mathrm{TN}, \mathrm{WV}$ ) recruited and delivered 6 sessions every two weeks that focused on cooking skills, family mealtime, and increasing physical activity. The Peds-QoL tool assessed perceived QoL in youth and adults related to their functioning physically, emotionally, and socially.

Outcome Measures and Analysis: Nested f-tests and additive linear mixed modeling.

Results: Compared to a two-way interaction model (pvalue 0.6052$)$ the three-way model became close to significant (p-value 0.0547) in youth SBP interaction with group, time and PEDS QoL. Compared to the additive statistical model in adult SBP, the two-way simple statistical model wasn't a better predictor using group, time and PED QoL to show significance (p-value 0.1691 vs 0.1108 respectively).

Conclusions and Implications: Using simple and sophisticated statistical analysis to understand interaction and response to SBP, between treatment and control groups, over time and with perceived PED QoL was not

Continued on page S84 